psychosocial health, but it is not clear what components of OA disease severity are related to depression onset in these patients.

Objectives: The aim was to determine the components of OA disease severity that contribute to depression onset among individuals with radiographic knee OA. Methods: Eligible participants $(n=1,652)$ were from the Osteoarthritis Initiative with radiographic disease (Kellgren-Lawrence [K-L] grade 2, 3, or 4), complete baseline covariate data, and were below the screening threshold for probable depression (Centre for Epidemiological Studies Depression [CES-D] Scale $\leq 16$ ). OA disease severity was assessed at baseline and three annual follow-up visits using minimum joint space width (millimetres), 20-metre gait speed (metres per second), and pain subscale of the Western Ontario and McMaster Universities Osteoarthritis Index (WOMAC). OA disease severity predictors were evaluated as a moving average at each time point then categorised in quintiles. Joint space width and gait speed categories were inverted so that increasing quintile corresponded to greater disease severity. Depression onset was assessed at four annual follow-up visits using the CES-D. Potential confounders included timeinvariant and time-varying measures: demographics, lifestyle factors, socioeconomic status, Charlson comorbidity index, K-L grade, and WOMAC functional disability and joint stiffness; and CES-D score, body mass index, analgesic use, and knee injuries, respectively. Marginal structural models that account for timedependent confounding and selective attrition modelled the association between each time-varying disease severity predictor and depression onset.

Results: There was a non-linear probability (figure 1) for onset of depression by disease severity quintiles for the three predictors. Moreover, the risk of depression was greatest in the highest severity quintiles and reached statistical significance for all three predictors. Odd ratios comparing highest to lowest severity quintiles in order of increasing magnitude were 1.80 (95\% confidence interval [Cl]: 1.00, 3.24) for gait speed, 2.10 (95\% Cl: 1.17, 3.75) for joint space width, and 2.21 (95\% Cl: $1.14,4.30)$ for pain.

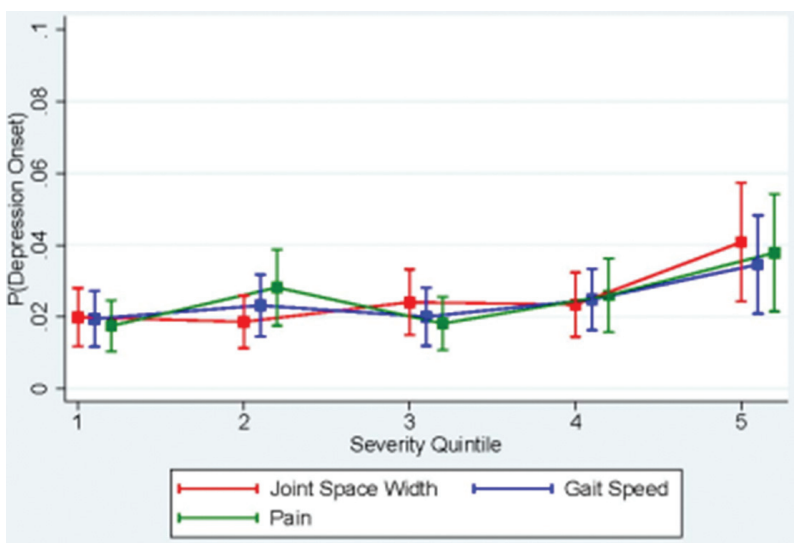

Conclusions: Findings demonstrate that the presence of greater structural disease severity and pain and decreased physical performance increases the risk of depression onset among individuals with radiographic knee OA. Thus, it is necessary to also intervene on OA disease severity when treating depression to effectively manage these patients. A combined treatment strategy consisting of two interventions delivered in parallel to simultaneously treat each condition may be the most effective form of medical care for OA patients who have comorbid depression.

Acknowledgements: This study was supported by the Rheumatology Research Foundation's Scientist Development Award.

Disclosure of Interest: A. Rathbun Grant/research support from: Rheumatology Research Foundation, Z. Evans: None declared, M. Shardell: None declared, M. Yau: None declared, J. Gallo: None declared, E. Stuart: None declared, M. Schuler: None declared, M. Hochberg: None declared

DOI: 10.1136/annrheumdis-2018-eular.7224

\section{OP0004 COGNITIVE IMPAIRMENT IN RHEUMATOID ARTHRITISPATIENTS: A CASE-CONTROL STUDY}

B.K. Vitturi ${ }^{1}$, B.A.C. Nascimento ${ }^{2}$, B.R. Alves ${ }^{2}$, F. Campos ${ }^{2}$, D.Y. Torigoe ${ }^{2}$. ${ }^{1}$ Division of Neurology; ${ }^{2}$ Division of Rheumatology, Irmandade da Santa Casa de Misericórdia de São Paulo, Sao Paulo, Brazil

Background: Rheumatoid arthritis (RA) is the most common autoimmune inflammatory arthritis in adults. ${ }^{1}$ Extra-articular manifestations of RA can occur in about $40 \%$ of patients, either in the beginning or during the course of their disease. ${ }^{2}$ Recent studies have suggested that RA may have an important relation in the development of cognitive and neurological dysfunction. ${ }^{3}$ However, the bond between RA and the brain is still uncertain.

Objectives: The purpose of this study was to assess the frequency and the clinical predictors of cognitive impairment in rheumatoid arthritis (RA) patients.

Methods: A cross-sectional and case-control study was performed including consecutive RA patients seen in a rheumatology outpatient clinic of referral tertiary hospital. The control group included 100 healthy subjects. We registered clinical and demographic data including age, sex, level of education, time of disease, time of diagnosis, drugs in use, cardiovascular risk factors and other comorbidities. Functional capacity was assessed using the Health Assessment Questionnaire (HAQ). Neurological appraisal was made with standardised questionnaires: MiniMental State Examination (MMSE), Montreal Cognitive Assessment (MoCA) and the Hospital Anxiety and Depression (HAD). Analysis of the data was performed using qui-square and t-tests and a multivariate analysis (SPSS 22.0). Significance level was set as $<0.05$.

Results: We included 102 patients (88 were female) with a mean age of 57.4 $( \pm 10.7)$ years; among these patients, $92(90.2 \%)$ used corticosteroids, $66(64.7 \%)$ methotrexate, $5(4.9 \%)$ sulfasalazine, $43(42.1 \%)$ leflunomide, $18(17.6 \%)$ chloroquine and $25(24.5 \%)$ biologic therapy. The mean age of RA diagnosis was 42.9 $( \pm 12.7)$ years. In both univariate and multivariate analysis, compared to the control group, patients with RA presented significant lower MMSE $(22.5 \pm 4.0)$ and MoCA (17.1 \pm 4.5$)$ scores $(p<0.05)$. Adjusting for level of education, just 31 and 25 patients presented normal MMSE and MoCA scores $(p<0.01)$, respectively. Cognitive decline was associated with higher $\mathrm{HAQ}$ scores (functional outcome due to $\mathrm{RA})$ and prolonged time of disease $(\mathrm{p}<0.05)$. No correlation was found between sex, disease-modifying antirheumatic drugs, rheumatoid factor, C-reactive protein levels and the neurological impairment. The mean HAD score was $17.2( \pm 8.2)$ and anxiety and depression were more prevalent in RA patients than in control group $(p<0.01)$

Conclusions: Patients with rheumatoid arthritis may present with cognitive decline and dementia as extra-articular manifestations of the disease. Neurological impairment is usually disregarded and might be under-diagnosed in RA patients. Future studies are necessary in order to better understand the relationship between RA and the Brain.

\section{REFERENCES:}

[1] Helmick CG, Felson DT, Lawrence RC, Gabriel S, Hirsch R, Kwoh CK, et al. Estimates of the prevalence of arthritis and other rheumatic conditions in the United States: part I. Arthritis Rheum 2008;58:15-25.

[2] Pincus T, Callahan LF, Sale WG, Brooks AL, Payne LE, Vaughn WK Severe functional declines, work disability, and increased mortality in seventy-five rheumatoid arthritis patients studied over nine years. Arthritis Rheum1984;27:864-72.

[3] Gimeno D, Marmot MG, Singh-Manoux A. Inflammatory markers and cognitive function inmiddle-aged adults: The Whitehall II study. Psychoneuroendocrinology 2008;33:1322-34.

Disclosure of Interest: None declared

DOI: 10.1136/annrheumdis-2018-eular.6685

WEDNESDAY, 13 JUNE 2018

Shaping the future in systemic sclerosis

\begin{tabular}{|l|l}
\hline OP0005 & PATTERNS OF 31 NEW AUTOANTIBODIES AGAINST G \\
PROTEIN-COUPLED RECEPTORS AND GROWTH \\
FACTORS IN SYSTEMIC SCLEROSIS CAN BE \\
DESCRIBED BY LATENT FACTORS
\end{tabular}

H. Bittern ${ }^{1}$, A. Carvalho-Marques ${ }^{1}$, O. Cabral-Marques ${ }^{1}$, C. Fouodo², I. König², H. Heidecke ${ }^{3}$, G. Riemekasten ${ }^{1}$, S. Schinke ${ }^{1}{ }^{1}$ Rheumatology and Clinical Immunology UKSH Lübeck, ${ }^{2}$ Institute of Medical Biometry and Statistics, -, Lübeck; ${ }^{3}$ CellTrend GmbH, Biotechnology, Luckenwalde, Germany

Background: Systemic sclerosis (SSc) is a rare autoimmune multisystemic disease with a significant disease burden and impact on life quality and survival. Disease specific, diagnostic and prognostic antibodies $(a b)$ are known such as Scl70 and centromer (ACA) $a b^{1}$ or recently endothelin or angiotensin receptors. ${ }^{2}$ Functional ab can bind G protein-coupled receptors (GPCR) regulating immune function and were reported in the pathogenesis of various inflammatory and noninflammatory diseases. ${ }^{3}$

Objectives: We analysed $31 \mathrm{ab}$ against GPCRs and growth factors in a retrospective cohort of 71 SSc patients compared to 196 sera from healthy controls $(\mathrm{HC})$. Ab levels were related to disease manifestations such as sex, age, SSc phenotype in order to hypothesise functional ab and new pathogenic targets in SSc. 
Methods: The retrospective clinical characterisation of 14 male and 57 female SSc patients (26-82 years) included mRSS, organ involvement assessed by laboratory tests, spirometry and imaging such as CT-scan or echocardiography. 30/ 71 had active disease (EUSTAR activity score). Ab were measured by ELISA and normalised to a standard serum. Median ab levels from SSc were compared to HC (Mann Whitney Test). Ab patterns were analysed using different statistical approaches (factor analysis, principal component analysis (PCA), linear discriminant analysis (LDA), cluster analysis and biserial correlation.

Results: Clinical SSc subgroups (diffuse/limited cutaneous, male/female) differ in ab levels and form separate clusters (LDA method). Moreover, 5 resp. 7 latent factors group $a b$ and clinical disease manifestations. Factor analysis reveals VEGFR2 and YBX1 ab to be more unique with the lowest communalities. The biserial correlation shows moderate associations between ab patterns and SSc specific symptoms such as Raynaud's, calcinosis or akroosteolysis but also unspecific symptoms such as polyneuropathy. Compared to association of ETAR ab with Raynaud's and skin sclerosis HGFR ab are inversely correlated. In HC most ab levels against GPCR and growth factors are higher than in SSc except for YBX1 which has the highest ab levels in SSc patients. In $\mathrm{HC}$ ab levels against YBX1 ab are associated with male sex and family history of rheumatic diseases. Yet, ADRB2 ab are linked to the absence of Gl symptoms or depression and $a b$ against ENG, ETAR, PAR2, PAR1 with normal troponine levels (absence of heart involvement).
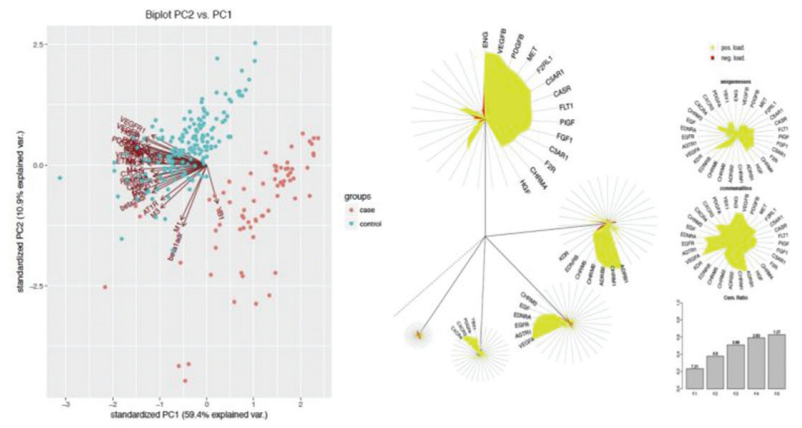

Abstract OP0005 - Figure 1. (a) Projection of individuals (points) and variables (arrows) representing the measured proteins, in the space of the two principle components. The almost perfect separation between controls (cyan) and cases (orange) is explained by higher protein levels for almost all proteins in the HD groups against lower values in the SSc group. By contrast, only the YBI_P18 protein presents a tendance to be higher in the SSc group and lower in the other group. M1 and betaladr proteins have lowest contributions for separing HG from SSc participiants. (b) Dandelion plot displays 5 latent factors the cluster antibodies against GPCR and growth factors in SSc

Conclusions: We describe 31 new ab against GPCR and growth factors in SSc. $\mathrm{Ab}$ as well as SSc disease manifestations could be clustered by latent factors Most ab titers in SSc were lower than in HC. Some ab were linked to the absence of SSc manifestations. Thus, we postulate that a dysbalance of functionally protective autoantibodies, that can be found in healthy individuals, and the appearance of SSc specific ab such as Scl70 contribute to its pathogenesis. Considering the preliminary character of our data, the functional impact of ab against GPCR and growth factors has to be validated in vitro and statistical correlations to be confirmed in a prospective independent patient cohort.

\section{REFERENCES:}

[1] Herrick AL, et al. $A R D$ 2018(0):1-8.

[2] Kill A, et al. Curr Rheumatol Rep 2015;(17):34

[3] Cabral-Marques O, et al. Nat. Rev 2017;(13):648-656.

Disclosure of Interest: None declared

DOI: 10.1136/annrheumdis-2018-eular.2711

\section{OP0006 \\ SAFETY AND EFFICACY OF LENABASUM (JBT-101) IN DIFFUSE CUTANEOUS SYSTEMIC SCLEROSIS SUBJECTS TREATED FOR ONE YEAR IN AN OPEN- LABEL EXTENSION OF TRIAL JBT101-SSC-001}

R. Spiera ${ }^{1}$, L. Hummers ${ }^{2}$, L. Chung ${ }^{3}$, T. Frech ${ }^{4}$, R. Domsic ${ }^{5}$, V. Hsu ${ }^{6}$, D.E. Furst ${ }^{7}$, J. Gordon ${ }^{1}$, M. Mayes ${ }^{8}$, R. Simms ${ }^{9}$, E. Lee ${ }^{10}$, N. Dgetluck $^{10}$, S. Constantine ${ }^{10}$, B. White ${ }^{10}$. ${ }^{1}$ Hospital for Special Surgery, New York City; ${ }^{2}$ Johns Hopkins,

Baltimore; ${ }^{3}$ Stanford University, Palo Alto; ${ }^{4}$ University of Utah, Salt Lake City,

${ }^{5}$ University of Pittsburgh, Pittsburgh; ${ }^{6}$ Rutgers Robert Wood Johnson Medical School, New Brunswick; ${ }^{7}$ Arthritis Association of Southern California, Los Angeles; ${ }^{8}$ University of Texas, Houston; ${ }^{9}$ Boston University, Boston; ${ }^{10}$ Corbus Pharmaceuticals, Inc., Norwood, USA

Background: Lenabasum (JBT-101) is a selective cannabinoid receptor type 2 agonist that activates resolution of innate immune responses in humans and reduces inflammation and fibrosis in animal models of SSc. It is a synthetic, oral, non-immunosuppressive small molecule. Lenabasum had acceptable safety and tolerability and showed evidence of clinical benefit in diffuse cutaneous SSc (dcSSc) in Phase 2 trial JBT101-SSc-001 (NCT02465437).

Objectives: The objective of this study was to provide long-term open-labe safety and efficacy data in dcSSc subjects who received lenabasum in that trial. Methods: Subjects who completed the double-blind placebo-controlled (DBPC) part of JBT101-SSc-001 were eligible to receive lenabasum $20 \mathrm{mg}$ BID in an open-label extension (OLE).

Results: $36 / 38$ (95\%) eligible subjects enrolled in the OLE and 34/36 (94\%) were on baseline immunosuppressive drugs. The mean interval off study drug from the end of DBCP dosing to the start of OLE dosing of 9.5 weeks (range 4.7 to 56 weeks). At the time of data cut-off, the duration of OLE dosing was median 51.4 weeks, mean 45 weeks (range 26, 418 weeks), and 19 subjects had completed Week 60. Three subjects discontinued, 2 for AEs and 1 for withdrawal of consent. Adverse events (AEs, $n=171$ ) occurred in $33 / 36(92 \%)$ subjects in the OLE. By maximum severity, $1(3 \%)$ subject had life threatening $A E, 3(8 \%)$ subjects had severe AEs, $21(58 \%)$ subjects had moderate AEs and $8(22 \%)$ had mild AEs. Seven (19\%) subjects had AEs considered related to lenabasum. The AEs that occurred in $\geq 10 \%$ of subjects ( $n, \%$ of subjects) were upper respiratory tract infection $(8,22 \%)$, urinary tract infection $(5,14 \%)$, diarrhoea $(4,11 \%)$ and skin ulcers $(4,11 \%)$. Mild intermittent dizziness occurred in $3(8 \%)$ subjects. One subject developed renal crisis 7 days after starting $60 \mathrm{mg} /$ day prednisone prescribed by a non-study physician and had 2 severe and 1 life-threatening/serious AEs related to the renal crisis and deemed unrelated to lenabasum. During the OLE, there was improvement in multiple efficacy outcomes from both the study start and the OLE start. For example, in the 25 subjects who had completed OLE Week 52 at the time of data cut-off, the mean (SE) improvements from study start were: ACR CRISS score=56\% (9\%); modified Rodnan Skin Score=-8.6 (1.5); HAQ-DI=-0.14 (0.11), Physician Global Assessment=-0.9 (0.5), and 5-D Itch Questionnaire $=-2.3$ (0.8). Forced vital capacity\% predicted was stable from study start with mean (SE) change $=0.4 \%(0.7 \%)$.

Conclusions: In OLE of Phase 2 trial JBT101-SSc-001, lenabasum continues to have acceptable safety and tolerability in dcSSc with no severe or serious AEs. Multiple efficacy outcomes improved, although open-label nature of dosing with lenabasum is acknowledged. These data support Phase 3 testing of lenabasum for treatment of dcSSc.

Disclosure of Interest: R. Spiera: None declared, L. Hummers: None declared L. Chung: None declared, T. Frech: None declared, R. Domsic: None declared, V. Hsu: None declared, D. Furst: None declared, J. Gordon: None declared, M. Mayes Consultant for: Boehringer-Ingelheim, Mitsubishi-Tanabe, Astellas Roche-Genentech, R. Simms: None declared, E. Lee Employee of: Corbus Pharmaceuticals, Inc., N. Dgetluck Employee of: Corbus Pharmaceuticals, Inc., S. Constantine Employee of: Corbus Pharmaceuticals, Inc., B. White Employee of: Corbus Pharmaceuticals, Inc.

DOI: 10.1136/annrheumdis-2018-eular.3512

WEDNESDAY, 13 JUNE 2018

\section{Basic mechanisms of inflammation} \begin{tabular}{|l|l}
\hline OP0007 & GROWTH DIFFERENTIATION FACTOR 11 ATTENUATES \\
INFLAMATORY ARTHRITIS THROUGH ANTAGONISING
\end{tabular} NF-KB SIGNALLING PATHWAY

Y. Zhao ${ }^{1}$, W. Liं ${ }^{2}, \mathrm{~K}$. Vasilev ${ }^{3} .{ }^{1}$ Department of Orthopaedics; ${ }^{2}$ Department of Pathology, Shandong University Qilu Hospital, Jinan, China; ${ }^{3}$ School of engineering, University of South Australia, Mawson Lakes, Australia

Background: It is well established that the tumour necrosis factor- $\alpha$ (TNF- $\alpha$ ) plays a dominant role in rheumatoid arthritis (RA) ${ }^{1}$ and other arthritis models. ${ }^{2}{ }^{3}$ Growth Differentiation Factor 11 (GDF11) is recently reported to be closely 